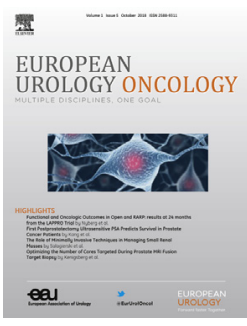

\title{
Systemic Inflammatory Markers and Oncologic Outcomes in Patients with High-risk Non-muscle-invasive Urothelial Bladder Cancer
}

\author{
Francesco Cantiello $^{a, 1}$, Giorgio I. Russo ${ }^{b, 1, *}$, Mihai Dorin Vartolomei ${ }^{c, d, e}$, \\ Abdal Rahman Abu Farhan ${ }^{a}$, Daniela Terracciano ${ }^{f}$, Gennaro Musi ${ }^{c}$, Giuseppe Lucarelli ${ }^{g}$, \\ Savino M. Di Stasi ${ }^{h}$, Rodolfo Hurle ${ }^{i}$, Vincenzo Serretta ${ }^{j}$, Gian Maria Busetto ${ }^{k}$, Chiara Scafuro $^{a}$, \\ Sisto Perdona ${ }^{l}$, Marco Borghesi ${ }^{m}$, Riccardo Schiavina ${ }^{m}$, Antonio Cioffi ${ }^{c}$, Ettore De Berardinis ${ }^{n}$, \\ Gilberto L. Almeida ${ }^{o}$, Pierluigi Bove ${ }^{i}$, Estevao Lima ${ }^{p}$, Giuseppe Ucciero ${ }^{a}$, Deliu Victor Matei ${ }^{c, q}$, \\ Nicolae Crisan ${ }^{q}$, Paolo Verze ${ }^{r}$, Michele Battaglia ${ }^{h}$, Giorgio Guazzoni ${ }^{j}$, Riccardo Autorino $^{s}$, \\ Giuseppe Morgia ${ }^{b}$, Rocco Damiano $^{a}$, Ottavio de Cobelli ${ }^{c}$, Vincenzo Mirone ${ }^{r}$, \\ Shahrokh F. Shariat ${ }^{t, u, v}$, Matteo Ferro ${ }^{c}$ \\ ${ }^{\mathrm{a}}$ Department of Urology, Magna Graecia University of Catanzaro, Catanzaro, Italy; ${ }^{\mathrm{b}}$ Department of Urology, University of Catania, Catania, Italy; ${ }^{\mathrm{c}}$ Division of \\ Urology, European Institute of Oncology, Milan, Italy; ${ }^{\mathrm{d}}$ Department of Urology, Medical University of Vienna, Vienna, Austria; ${ }^{\mathrm{e}}$ Department of Cell and \\ Molecular Biology, University of Medicine and Pharmacy, Tirgu Mures, Romania; ${ }^{\mathrm{f}}$ Department of Translational Medical Sciences, University of Naples \\ Federico II, Naples, Italy; ${ }^{\mathrm{g}}$ Department of Emergency and Organ Transplantation, Urology, Andrology and Kidney Transplantation Unit, University of Bari, \\ Bari, Italy; ${ }^{\mathrm{h}}$ Department of Surgery and Experimental Medicine, University Tor Vergata, Rome, Italy; ${ }^{\mathrm{i}}$ Department of Urology, Humanitas Hospital, Milan, \\ Italy; ${ }^{\mathrm{j}}$ Department of Urology, University of Palermo, Palermo, Italy; ${ }^{\mathrm{k}}$ Department of Urology, Sapienza University of Rome, Rome, Italy; ${ }^{1}$ Uro-Gynecological \\ Department, Istituto Nazionale per lo Studio e la Cura dei Tumori, Fondazione G. Pascale IRCCS, Naples, Italy; ${ }^{\mathrm{m}}$ Department of Urology, University of Bologna, \\ Bologna, Italy; ${ }^{\mathrm{n}}$ Department of Gynecological-Obstetrics Sciences and Urological Sciences, Sapienza Rome University Policlinico Umberto I, Rome, Italy; \\ ${ }^{\circ}$ Department of Urology, University of Vale do Itajaí, Itajaí, Brazil; ${ }^{\mathrm{p}}$ Department of CUF Urology and Life and Health Sciences Research Institute, University of \\ Minho, Braga, Portugal; ${ }^{\mathrm{q}}$ Department of Urology, University of Medicine and Pharmacy Iuliu Haieganu, Cluj-Napoca, Romania; ${ }^{\mathrm{r}}$ Department of \\ Neurosciences, Sciences of Reproduction and Odontostomatology, Urology Unit, University of Naples Federico II, Naples, Italy; ${ }^{\mathrm{s}}$ Division of Urology, Virginia \\ Commonwealth University, Richmond, VA, USA; ${ }^{\mathrm{t}}$ Karl Landsteiner Institute of Urology and Andrology, Vienna, Austria; ${ }^{\mathrm{u}}$ Department of Urology, University of \\ Texas Southwestern Medical Center, Dallas, TX, USA; ${ }^{2}$ Department of Urology, Weill Cornell Medical College, New York, NY, USA
}

\section{Article info}

Article history:

Accepted June 12, 2018

Associate Editor:

Ashish Kamat

Keywords:

Bladder cancer

Neutrophil/lymphocyte ratio

Platelet/lymphocyte ratio

Lymphocyte/monocyte ratio

Prognosis

\begin{abstract}
Background: Serum levels of neutrophils, platelets, and lymphocytes have been recognized as factors related to poor prognosis for many solid tumors, including bladder cancer (BC).

Objective: To evaluate the prognostic role of the combination of the neutrophil/ lymphocyte ratio (NLR), platelet/lymphocyte ratio (PLR), and lymphocyte/monocyte ratio (LMR) in patients with high-risk non-muscle-invasive urothelial BC (NIMBC). Design, setting, and participants: A total of 1151 NMIBC patients who underwent first transurethral resection of the bladder tumor (TURBT) at 13 academic institutions between January 1, 2002 and December 31, 2012 were included in this analysis. The median follow-up was 48 mo.

Intervention: TURBT with intravesical chemotherapy or immunotherapy.

\footnotetext{
${ }^{1}$ These authors equally contributed to this paper.

* Corresponding author. Urology Section, University of Catania, Via Santa Sofia 78, Catania, Italy. Tel.: +39095 3782710 .

E-mail address: giorgioivan1987@gmail.com (G.I. Russo).
} 
Outcome measurements and statistical analysis: Multivariable Cox regression analysis was performed to identify factors predictive of recurrence, progression, cancer-specific mortality, and overall mortality. A systemic inflammatory marker (SIM) score was calculated based on cutoffs for NLR, PLR, and LMR.

Results and limitations: The 48-mo recurrence-free survival was 80.8\%, 47.35\%, $20.67 \%$, and $17.06 \%$ for patients with an SIM score of $0,1,2$, and 3, respectively ( $p<0.01$, log-rank test) while the corresponding 48-mo progression free-survival was $92.0 \%, 75.67 \%, 72.85 \%$, and $63.1 \%$ ( $p<0.01$, log-rank test). SIM scores of 1,2 , and 3 were associated with recurrence (hazard ratio [HR] 3.73, 7.06, and 7.88) and progression (HR 3.15, 4.41, and 5.83). Limitations include the lack of external validation and comparison to other clinical risk models.

Conclusions: Patients with high-grade T1 stage NMIBC with high SIM scores have worse oncologic outcomes in terms of recurrence and progression. Further studies should be conducted to stratify patients according to SIM scores to identify individuals who might benefit from early cystectomy.

Patient summary: In this study, we defined a risk score (the SIM score) based on the measurement of routine systemic inflammatory markers. This score can identify patients with high-grade bladder cancer not invading the muscular layer who are more likely to suffer from tumor recurrence and progression. Therefore, the score could be used to select patients who might benefit from early bladder removal.

(C) 2018 European Association of Urology. Published by Elsevier B.V. All rights reserved.

\section{Introduction}

Neutrophils act as short-lived effector cells of the innate immune system and play a primary role in resistance against extracellular pathogens and inflammation [1]. The tumor microenvironment is characterized by stimulation of the immune system, which leads to increases in various host components including stromal cells, growing blood vessels, and inflammatory infiltrates [1,2]. All these components have a pivotal role in the development and progression of many malignancies, including bladder cancer (BC) [3], through the release of cytokines by the tumor microenvironment [4].

Many biomarkers from routine blood tests have been evaluated in predicting BC outcomes in recent years, including hemoglobin levels, platelet counts, leukocyte counts, and C-reactive protein (CRP). In this regard, neutrophils and lymphocytes have been combined in the neutrophil/lymphocyte ratio (NLR). A high NLR has been recognized as a factor related to poor prognosis for many solid tumors, including $\mathrm{BC}$ [3]. A recent metaanalysis by Tang et al [5] proved that NLR had a predictive value for survival outcomes in BC patients, and that patients with a higher pretreatment NLR experienced worse survival.

Moreover, the systemic inflammatory response to tumors is associated with abnormalities of several blood components, including neutrophils, lymphocytes, and platelets [6]. However, the role of systemic inflammatory markers has been not fully investigated in the context of BC. In fact, current prognostic models for non-muscle-invasive bladder cancer (NMIBC) relying on pathological features such as T stage, grade, focality, tumor diameter, recurrence rate and concomitant carcinoma in situ (CIS) do not reach included these kinds of markers that could play a significant prognostic role [7].

The aim of this multicenter study was to evaluate the prognostic role of systemic markers of inflammation among patients with high-risk NMIBC.

\section{Patients and methods}

\subsection{Patient population}

A total of 1155 high-risk NMIBC patients treated at 13 academic institutions between January 1, 2002 and December 31, 2012 were included in this prospective observational study. Institutional review board approval was obtained at the leading center (IEO, Milan, Italy), and a data usage agreement was established with all participating centers.

Demographic, clinical and pathologic data for first and second transurethral resection of bladder tumor (TURBT) were collected for each patient and entered in a purpose-built database. Tumor histology was assessed at each institution and classified using the American Joint Committee on Cancer (AJCC) staging manual (6th and 7th editions).

Second TURBT (re-TURBT) was performed by the same urologist who performed the first TURBT within $6 \mathrm{wk}$ [8]. The re-TURBT protocol included resection of the tumor scar and base, together with the bladder neck (for CIS) and red bladder patches. Each patient received adjuvant intravesical bacillus Calmette-Guérin (BCG) immunotherapy according to the European Association of Urology (EAU) recommendations [9]. The upper urinary tract was evaluated via radiological imaging in all subjects to exclude the presence of concomitant carcinoma. 
Laboratory parameters were measured $30 \mathrm{~d}$ before surgery and data for calculating the NLR, platelet/lymphocyte ratio (PLR), and lymphocyte/monocyte ratio (LMR) were collected. Patients with a urinary tract infection or inflammatory systemic diseases that could influence these parameters (chronic intestinal diseases, autoimmune diseases, leukaemia, lymphoma) were excluded from the study.

\subsection{Follow-up}

Only patients with $>3 \mathrm{yr}$ of follow-up were included. Follow-up was conducted according to EAU guidelines, with cystoscopy and urinary cytology every 3 mo [10]. The following oncologic outcomes were measured: time to first recurrence, time to first progression, cancer-specific mortality (CSM) and overall mortality (OM). Primary recurrence was defined as the reappearance of high-risk disease (high grade, T1, or CIS) after TURBT. Secondary progression was defined as development or a staging increase to lamina propria invasion (eg, increase from Ta to T1 or CIS to T1), muscle-invasive disease (stage $\geq \mathrm{T} 2$ ), lymph node $(\mathrm{N}+)$ or distant metastases (M1), or a grade increase from low to high (including CIS). CSM was defined as the time from random assignment to death resulting from bladder cancer, and $\mathrm{OM}$ as the time from random assignment to death resulting from any cause [9].

\subsection{Statistical analysis}

Continuous variables are presented as the median and interquartile range (IQR). Differences between groups were assessed using a Kruskall-Wallis or Mann-Whitney U test as appropriate. Categorical variables were tested using a $\chi^{2}$ test or Fisher's exact test.

Multivariable Cox regression analysis was performed to identify factors predictive of recurrence, progression, CSM, and OM using the variables measured. For internal validation, the analysis was subjected to 500 bootstrap resamples.

NLR, PLR, and LMR were dichotomized according to cutoffs already used in the literature, namely $2.5,150$, and 3.41, respectively [11-13]. A systemic inflammatory marker (SIM) score was calculated on the basis of positivity according to these cutoffs, with 1 point assigned for each value exceeding the specified threshold and then summed, yielding a final score ranging from 0 to 3 .

Kaplan-Meier curves were plotted to calculate the association between SIM and recurrence-free survival, progression-free survival, cancer-specific survival (CSS), and overall survival (OS). A log-rank test was used to verify statistical significance among the curves. Decision curve analysis (DCA) was performed to evaluate net benefit models included in the multivariable Cox regression analysis for predicting recurrence and progression. Subgroup analysis was performed according to smoking status.

All statistical analyses were carried out using Stata v.14 (StataCorp, College Station, TX, USA). For all comparisons, the significance level was set to $p<0.05$ for differences between groups.
Table 1 - Baseline characteristics of the study cohort $(n=1155)$

\begin{tabular}{|c|c|}
\hline Variable & Result \\
\hline Median age, yr (IQR) & $71.0(65.0-78.0)$ \\
\hline \multicolumn{2}{|l|}{ Gender, $n(\%)$} \\
\hline Male & $957(82.86)$ \\
\hline Female & $198(17.14)$ \\
\hline \multicolumn{2}{|l|}{ Smoking status, $n(\%)$} \\
\hline Never & $548(47.45)$ \\
\hline Current & $327(28.31)$ \\
\hline Former & $280(24.24)$ \\
\hline Median cigarettes per day, $n$ (IQR) & $10.0(0.0-20.0)$ \\
\hline Median smoking years, $n(\mathrm{IQR})$ & $20.0(0.0-30.0)$ \\
\hline Multifocal disease, $n(\%)$ & $516(44.68)$ \\
\hline Tumor size $\geq 3 \mathrm{~cm}, n(\%)$ & $746(64.64)$ \\
\hline Carcinoma in situ, $n(\%)$ & $156(13.51)$ \\
\hline Median erythrocyte sedimentation rate, $\mathrm{mm} / \mathrm{h}$ (IQR) & $13.0(8.0-22.0)$ \\
\hline Median C-reactive protein, mg/l (IQR) & $2.1(0.9-4.6)$ \\
\hline Median neutrophils, $\times 10^{3} / \mu \mathrm{l}(\mathrm{IQR})$ & $5.5(3.8-9.8)$ \\
\hline Median lymphocytes, $\times 10^{3} / \mu \mathrm{l}(\mathrm{IQR})$ & $1.92(1.5-2.6)$ \\
\hline Median monocytes, $\times 10^{3} / \mu \mathrm{l}(\mathrm{IQR})$ & $0.53(0.40-0.76)$ \\
\hline Median eosinophils, $\times 10^{3} / \mu \mathrm{l}(\mathrm{IQR})$ & $0.17(0.10-0.28)$ \\
\hline 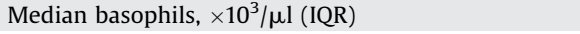 & $0.03(0.01-0.05)$ \\
\hline Median platelets, $\times 10^{3} / \mu \mathrm{l}(\mathrm{IQR})$ & $233.0(193.0-283.0)$ \\
\hline Median neutrophil/lymphocyte ratio (IQR) & $3.0(1.95-4.58)$ \\
\hline Median platelet/lymphocyte ratio (IQR) & $119.7(85.91-163.16)$ \\
\hline Median lymphocyte/monocyte ratio (IQR) & $3.5(2.4-5.22)$ \\
\hline
\end{tabular}

\section{Results}

\subsection{Baseline characteristics in the overall study population}

Table 1 lists the baseline characteristics of the study cohort. The median age was $71.0 \mathrm{yr}$ (IQR 65.0-78.0), median NLR was 3.0 (IQR 1.96-4.6), median PLR was 119.7 (IQR 85.9-163.2), median LMR was 3.5 (IQR 2.4-5.2), and median SIM was 2 (IQR 1-2). Overall, 221 patients (19.1\%) had previously received chemotherapy instillation: 53 (4.6\%) received epirubicin and 168 (14.5\%) mitomycin $40 \mathrm{mg}$. After re-TURBT, 288 patients $(24.9 \%)$ had residual high-grade NMIBC, while 867 (75.1\%) were negative. All patients received BCG immunotherapy and the median regimen duration was 12.0 mo (IQR 6.0-36.0). When patients were dichotomized according to the defined cutoffs, 778 (67.4\%), 344 (29.8\%), and 597 (51.7\%) had NLR $\geq 2.5$, PLR $\geq 150$, and LMR $\geq 3.41$, respectively.

\subsection{Clinical and pathologic characteristics according to marker cutoffs}

Table 2 shows the clinical and pathologic characteristics according to the previously defined marker cutoffs. Patients with NLR $\geq 2.5$ had a significantly higher erythrocyte sedimentation rate (ESR; 15.0 vs $12.0 \mathrm{~mm} / \mathrm{h} ; p<0.01$ ) and CRP (2.3 vs $2.0 \mathrm{mg} / \mathrm{l} ; p=0.02$ ). Similarly, for patients with $\mathrm{LMR} \geq 3.41$, both ESR (15.0 vs $12.0 \mathrm{~mm} / \mathrm{h} ; p<0.01$ ) and CRP (2.9 vs $2.0 \mathrm{mg} / \mathrm{l} ; p<0.01$ ) were significantly higher. With regard to pathology, we found a higher rate of CIS only among patients with NLR $\geq 2.5$ ( $15.3 \%$ vs $9.8 \% ; p=0.011$ ) and a slower rate of tumor size $\geq 3 \mathrm{~cm}$ among patients with PLR $\geq 150$ (58.1\% vs 68.4\%; $p=0.003$ ). 


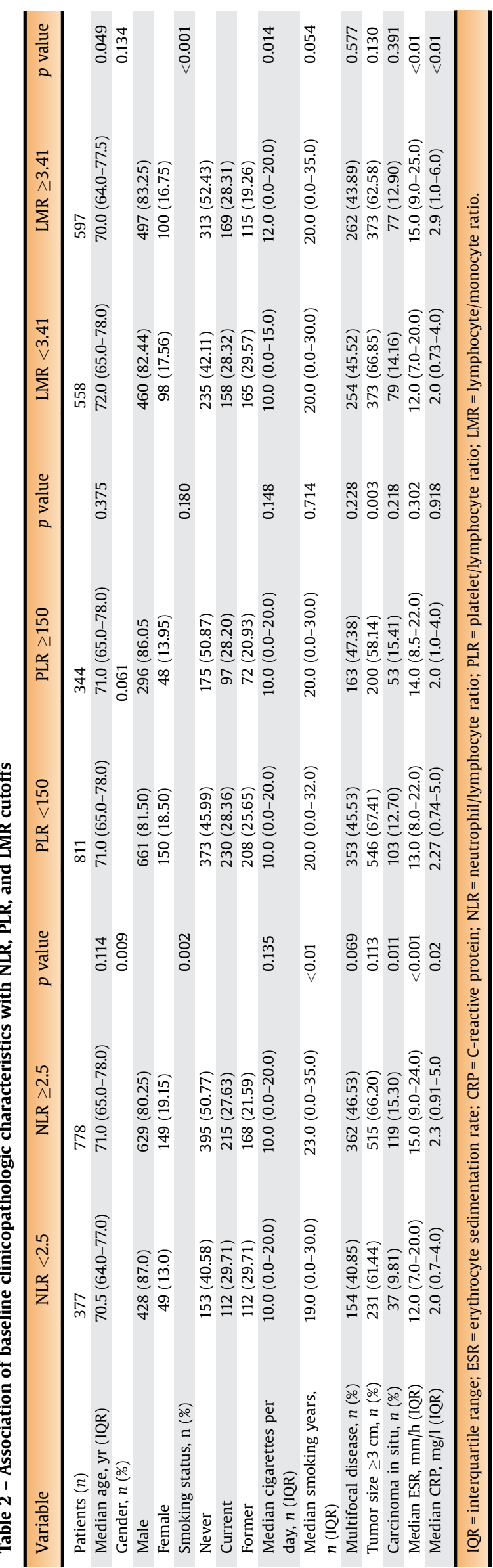

3.3. Clinical and pathologic characteristics according to SIM score

In total, 583 patients ( $50.5 \%$ ) had a SIM score $\geq 2$. Table 3 lists the clinical and pathologic variables according to SIM score. We found a significant increase in score with the number of cigarettes smoked and years of smoking. There was a lower rate of tumor size $\geq 3 \mathrm{~cm}$ among patients with high SIM score $(p=0.016)$, as well as a higher CIS rate $(p<0.01)$.

\subsection{Oncologic outcomes according to SIM score}

The median follow-up was 48 mo (IQR 40.0-66.0); in total, we observed 772 recurrences (66.8\%), 315 progressions (27.3\%), 81 cancer-specific deaths (7.0\%), and 162 overall deaths (14.0\%). The 48-mo recurrence-free survival was 80.8\% (95\% confidence interval [CI] 72.7-86.7\%), 47.3\% (95\% CI 42.4-52.1\%), 20.7\% (95\% CI 17.0-24.6\%), and 17.1\% (95\% CI 9.9-25.9\%) among patients with SIM scores of 0,1, 2, and 3, respectively ( $p<0.01, \log$-rank test). In multiple comparisons, between-group differences in recurrence-free survival were statistically significant (all $p<0.01$ ) except for 2 versus $3(p=0.24)$. The 48 -mo progression-free survival was 92.0\% (95\% CI 85.6-95.6\%), 75.7\% (95\% CI 70.6-80.0\%), 72.8\% (95\% CI 68.4-76.8\%), and 63.1\% (95\% CI 51.9-72.4\%) among patients with SIM scores of $0,1,2$, and 3, respectively ( $p<0.01$, log-rank test). In multiple comparisons, the between-group differences in progression free-survival were statistically significant (all $p<0.01$ ) except for 2 versus 3 ( $p=0.06$; Fig. 1$)$.

For the 48-mo CSS, we observed significant differences between SIM 0 and 3 (97.1\% vs 90.6\%; $p<0.01$ ), 1 and 3 (97.8\% vs 90.6\%; $p<0.01$ ), and 2 and 3 (94.4\% vs $90.6 \%$; $p=0.04)$. We observed significant differences in 48-mo OS only for SIM 1 versus 2 (93.9\% [95\% CI 90.7-96.0\%] and 89.6\% [95\% CI 85.9-92.4\%]; $p=0.02$; Fig. 2).

In bootstrap-adjusted multivariable Cox regression analyses, higher SIM score was associated with a significantly higher hazard ratio (HR) for recurrence (HR 3.7, 7.1, and 7.9) and progression (HR: 3.15, 4.4 and 5.8). Conversely, SIM was not associated with a higher probability of CSM or OM (Table 4).

Fig. 3 shows the DCA for models included in Table 4 and for individual factors. The model including SIM showed a greater clinical benefit of between 0.50 and 0.85 for predicting recurrence, and between 0.15 and 0.60 for progression when compared to all individual components.

\subsection{Oncologic outcomes according to smoking status}

We performed a subgroup analysis for current smokers and we found that the 48-mo recurrence-free survival was 65.0\% (95\% CI 48.2-77.6\%), 39.9\% (95\% CI 30.3-49.3\%), 19.6\% (95\% CI 13.1-27.1\%), and $16.2 \%$ (95\% CI 5.3-32.5\%) for patients with SIM scores $0,1,2$, and 3, respectively $(p<0.01)$. Multiple comparison revealed significant differences between SIM 0 and $1(p=0.04), 0$ and $2(p<0.01)$, 0 and $3(p<0.01), 1$ and $2(p<0.01)$, and 1 and $3(p<0.01$; Supplementary Fig. 1). 
Table 3 - Association between baseline clinicopathologic characteristics and cumulative marker score in the total cohort

\begin{tabular}{|c|c|c|c|c|c|}
\hline \multirow[t]{2}{*}{ Variable } & \multicolumn{4}{|c|}{ Systemic inflammatory marker score } & \multirow[t]{2}{*}{$p$ value } \\
\hline & 0 & 1 & 2 & 3 & \\
\hline Patients $(n)$ & 125 & 447 & 477 & 106 & \\
\hline Median age, yr (IQR) & $73(66.0-78.0)$ & $70.0(64.0) 77.0)$ & $71.0(65.0-78.0)$ & $70.0(67.0-79.0)$ & 0.13 \\
\hline \multicolumn{6}{|l|}{ Gender, $n(\%)$} \\
\hline Male & $10(86.40)$ & 365 (81.66) & $395(82.81)$ & 89 (83.69) & 0.648 \\
\hline Female & $17(13.60)$ & $82(18.34)$ & $82(17.19)$ & $17(16.04)$ & \\
\hline Smoking status, $n$ (\%) & & & & & $<0.001$ \\
\hline Never & $38(30.40)$ & $195(43.62)$ & $257(53.88)$ & $58(54.72)$ & \\
\hline Current & $40(32.00)$ & $125(27.96)$ & $130(27.25)$ & $32(30.19)$ & \\
\hline Former & $47(37.60)$ & $127(28.41)$ & $90(18.87)$ & $16(15.09)$ & \\
\hline Median cigarettes per day, $n$ (IQR) & $10.0(0.0-15.0)$ & $10.0(0.0-20.0)$ & $10.0(0.0-20.0)$ & $14.5(0.0-20.0)$ & 0.018 \\
\hline Median smoking years, $n(\mathrm{IQR})$ & $15.0(0.0-30.0)$ & $20.0(0.0-30.0)$ & $25.0(0.0-35.0)$ & $20.0(0.0-35.0)$ & $<0.01$ \\
\hline Multifocal disease, $n(\%)$ & $55(44.0)$ & $186(41.61)$ & $224(46.96)$ & $51(48.11)$ & 0.357 \\
\hline Tumor size $\geq 3 \mathrm{~cm}, n$ (\%) & $96(76.80)$ & $274(61.43)$ & $314(65.83)$ & $62(58.49)$ & 0.007 \\
\hline Carcinoma in situ, $n(\%)$ & $12(9.60)$ & $62(14.09)$ & $57(11.95)$ & $24(22.64)$ & 0.016 \\
\hline Median ESR, mm/h (IQR) & $8.0(5.8-14.0)$ & $13.0(8.0-20.0)$ & $15.0(10.0-25.0)$ & $16.0(8.0-25.0)$ & $<0.01$ \\
\hline Median CRP, mg/l (IQR) & $2.0(0.67-2.0)$ & $2.0(0.7-4.0)$ & $2.53(0.9-5.0)$ & $3.0(1.1-5.0)$ & $<0.01$ \\
\hline
\end{tabular}

A

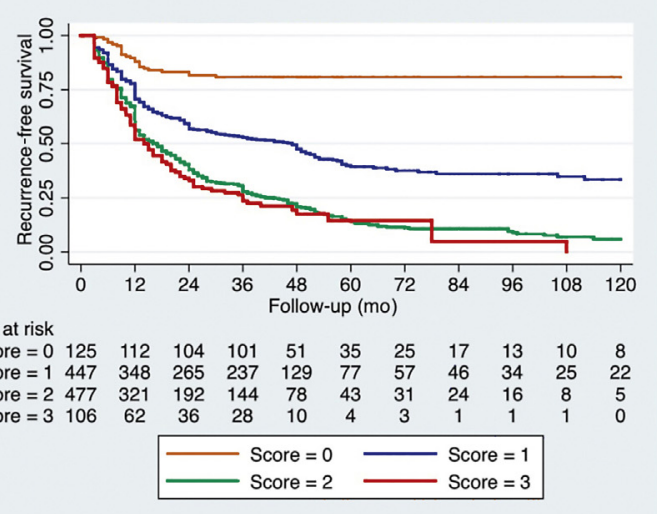

B

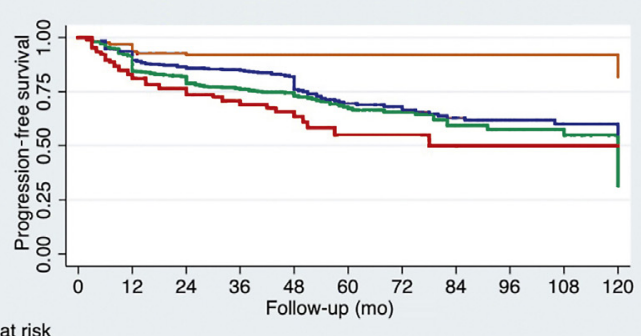

Number at risk

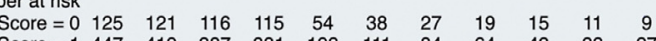

$\begin{array}{llllllllllll}\text { Score }=1 & 447 & 419 & 387 & 381 & 193 & 111 & 84 & 64 & 43 & 33 & 27\end{array}$

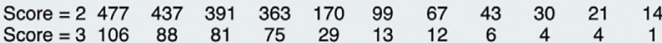

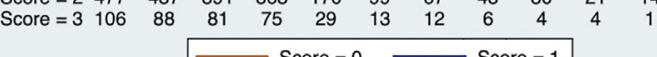

Fig. 1 - Kaplan-Meier estimates of (A) recurrence-free survival and (B) progression-free survival for high-grade T1 non-muscle-invasive bladder cancer stratified by systemic inflammatory marker score.

A

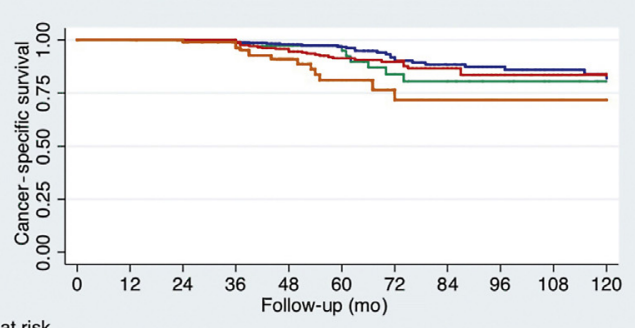

Number at risk

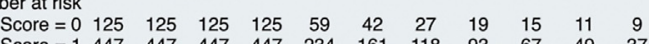

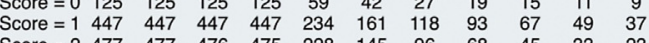

$\begin{array}{llllllllllll}\text { Score }=2 & 477 & 477 & 476 & 475 & 228 & 145 & 96 & 68 & 45 & 33 & 23 \\ \text { Score }=3 & 106 & 106 & 106 & 105 & 43 & 23 & 16 & 10 & 7 & 7 & 4\end{array}$

$\begin{array}{lllllll}- & \text { Score }=0 & & \end{array}$
B

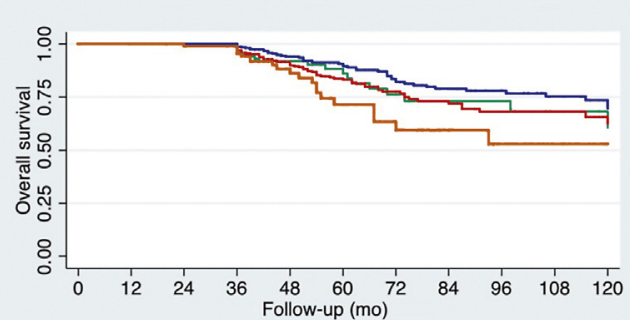

Number at risk

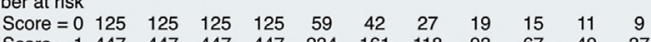

$\begin{array}{llllllllllll}\text { Score }=1 & 447 & 447 & 447 & 447 & 234 & 161 & 118 & 93 & 67 & 49 & 37\end{array}$ $\begin{array}{lccccccccccc}\text { Score }=2 & 477 & 477 & 476 & 475 & 228 & 145 & 96 & 68 & 45 & 33 & 23 \\ \text { Score }=3 & 106 & 106 & 106 & 105 & 43 & 23 & 16 & 10 & 7 & 7 & 4\end{array}$

Fig. 2 - Kaplan-Meier estimates of (A) cancer-specific survival and (B) overall survival for high-grade T1 non-muscle-invasive bladder cancer stratified by systemic inflammatory marker score. 
Table 4 - Multivariable Cox regression analysis for recurrence-free survival, progression-free survival, cancer-specific mortality, and overall mortality

\begin{tabular}{|c|c|c|c|c|c|c|c|c|}
\hline \multirow[t]{2}{*}{ Variable } & \multicolumn{2}{|c|}{ Recurrence-free survival } & \multicolumn{2}{|c|}{ Progression-free survival } & \multicolumn{2}{|c|}{ Cancer-specific mortality } & \multicolumn{2}{|c|}{ Overall mortality } \\
\hline & $\operatorname{HR}(95 \% \mathrm{CI})$ & $p$ value & $\operatorname{HR}(95 \% \mathrm{CI})$ & $p$ value & $\mathrm{HR}(95 \% \mathrm{CI})$ & $p$ value & $\mathrm{HR}(95 \% \mathrm{CI})$ & $p$ value \\
\hline Age in years & $1.00(0.99-1.01)$ & 0.99 & $0.99(0.98-1.00)$ & 0.309 & $1.03(1.01-1.06)$ & 0.006 & $1.05(1.02-1.06)$ & $<0.001$ \\
\hline \multicolumn{9}{|l|}{ Gender } \\
\hline Male & Reference & & Reference & & Reference & & Reference & \\
\hline Female & $1.15(0.96-1.38)$ & 0.130 & $1.20(0.91-1.58)$ & 0.194 & $0.88(0.49-1.59)$ & 0.686 & $1.02(0.68-1.52)$ & 0.923 \\
\hline \multicolumn{9}{|l|}{ Smoking status } \\
\hline Never & Reference & & Reference & & Reference & & Reference & \\
\hline Current & $1.01(0.86-1.20)$ & 0.86 & $0.84(0.62-1.12)$ & 0.24 & $0.92(0.49-1.76)$ & 0.803 & $1.06(0.70-1.60)$ & 0.428 \\
\hline Former & $0.69(0.57-0.84)$ & $<0.001$ & $1.32(1.02-1.71)$ & 0.036 & $1.25(0.75-2.05)$ & 0.383 & $0.85(0.59-1.23)$ & 0.382 \\
\hline Multifocal (yes vs no) & $1.22(1.05-1.40)$ & 0.007 & $1.31(1.05-1.64)$ & 0.016 & $1.17(0.75-1.82)$ & 0.469 & $1.13(0.83-1.55)$ & 0.428 \\
\hline $\begin{array}{l}\text { Tumor size } \\
(<3 \text { vs } \geq 3 \mathrm{~cm})\end{array}$ & $1.38(1.19-1.62)$ & $<0.001$ & $1.72(1.33-2.21)$ & $<0.001$ & $1.55(0.94-2.55)$ & 0.083 & $1.19(0.85-1.67)$ & 0.300 \\
\hline CIS (yes vs no) & $1.06(0.87-1.31)$ & 0.53 & $1.92(1.47-2.50)$ & $<0.001$ & $2.83(1.75-4.57)$ & $<0.001$ & $2.25(1.56-3.25)$ & $<0.001$ \\
\hline \multicolumn{9}{|l|}{ SIM score } \\
\hline 0 & Reference & & Reference & & Reference & & Reference & \\
\hline 1 & $3.73(2.45-5.68)$ & $<0.001$ & $3.15(1.69-5.86)$ & $<0.001$ & $0.68(0.31-1.47)$ & 0.328 & $0.36(0.37-1.08)$ & 0.099 \\
\hline 2 & 7.06 (4.67-10.69) & $<0.001$ & $4.41(2.38-8.18)$ & $<0.001$ & $1.00(0.47-2.13)$ & 0.981 & $0.97(0.58-1.62)$ & 0.909 \\
\hline 3 & $7.88(4.99-12.43)$ & $<0.001$ & $5.83(2.97-11.45)$ & $<0.001$ & $1.96(0.82-4.67)$ & 0.128 & $1.28(0.68-2.44)$ & 0.438 \\
\hline
\end{tabular}
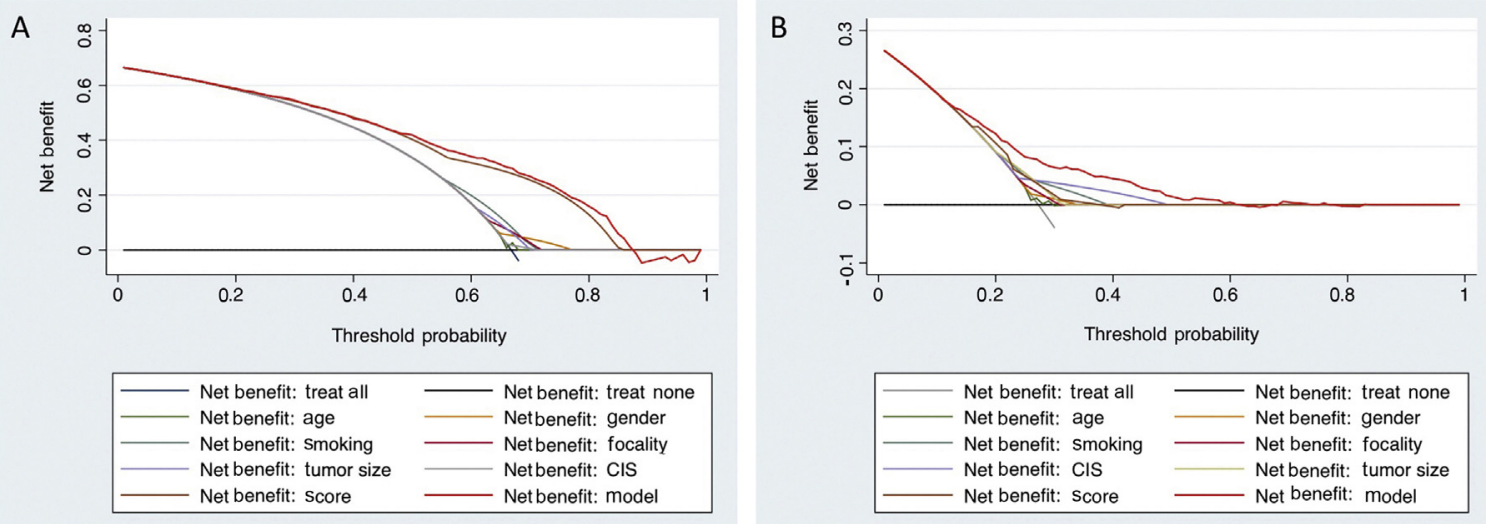

Fig. 3 - Decision curve analyses demonstrating the net benefit associated with (A) the recurrence and (B) the progression probabilities model. Decision curve analysis is a method for evaluating and comparing prediction models that incorporates clinical consequences.

Progression-free survival in this subgroup was $90.00 \%$ (95\% CI 75.51-96.12\%), 79.8\% (95\% CI 68.5-87.4\%), 78.2\% (95\% CI 69.6-84.6\%), and 63.9\% (95\% CI 40.3-80.2\%) for patients with SIM scores of $0,1,2$, and 3 , respectively $(p=0.09)$. Multiple comparisons revealed significnat differences between SIM 0 and $3(p=0.03)$ and 1 and $3(p=0.03)$.

\section{Discussion}

Our study aim was to investigate the predictive role of SIMs among patients with high-risk NMIBC. Several findings from our analysis are worthy of note. We found that a single score based on NLR, PLR, and LMR cutoffs, denoted as SIM, can predict recurrence and progression in this patient population. A higher SIM score is related to worse outcomes, even after adjusting for pathological variables such as tumor size, CIS, and multifocality. Furthermore, inclusion of this score in DCA seems to be of clinical benefit over individual components in the predictive model.

A growing body of evidence supports the role of inflammatory markers in urothelial cancers. Tanaka et al [14] showed that a score defined by preoperative NLR, plasma fibrinogen, and serum CRP was an independent predictor of patient survival following radical nephroureterectomy. The role of NLR was evaluated in a recent meta-analysis of 17 studies involving 3159 urinary cancer cases; elevated NLR predicted worse clinical outcomes. Subgroup analyses revealed that elevated NLR was associated with a high recurrence rate and poor cancer-specific survival in bladder cancer and urothelial carcinoma. Elevated NLR was a significant prognostic marker for worse recurrence-free survival and CSS, regardless of ethnic background, and 
predicted poor OS in the Caucasian population but not for Asian patients [15]. These results were confirmed by a study involving the Mayo Clinic Cystectomy Registry: patients with $\mathrm{NLR}=2.7$ had larger tumors (median 3.7 vs $2.6 \mathrm{~cm}$; $p<0.0001$ ) and were more likely at RC to have pathologic extravesical tumors (pT3/4; 44\% vs 32\%; $p<0.0001$ ), advanced nodal stage $(p=0.002)$, lymph node tumor involvement ( $20 \%$ vs $13 \% ; p=0.003$ ), a greater number of positive lymph nodes $(p=0.001)$, and lymph node density (7.4\% vs 3.4\%; $p=0.0008$ ). Likewise, elevated NLR was associated with subsequent disease recurrence, and cancerspecific and all-cause mortality [16].

Moreover, despite the inverse association between SIM and tumor size, our results show a significantly higher CIS rate among patients with higher SIM, strengthening its link with potential progression of the disease. Thus, the SIM score could serve in this setting in selecting patients who might benefit from an early radical cystectomy strategy. The current prognostic model for NMIBC, which relies on pathologic features such as T stage, grade, focality, tumor diameter, recurrence rate, and concomitant CIS, does not have sufficient accuracy in identifying such cases to ensure better survival [7].

$\mathrm{BC}$ should be considered a heterogeneous disease in which genes and epigenetic mutations, together with immune system alterations, play different roles. These considerations have increased interest in personalized, molecular targeting, and immunotherapeutic approaches to improve survival and overall prognosis among patients with BC [21]. In this context, it has been demonstrated that NLR is a biomarker of early response to targeted therapy among patients with metastatic renal cell carcinoma [17].

Our study is not devoid of limitations. First, our cohort may involve heterogeneity owing to the multi-institutional study design and the lack of central pathology review of TURB specimens. Second, laboratory assays and pathology examinations were performed separately at each institution without central review and we did not perform SIM measurement in a follow-up setting after TURB. However, as previously demonstrated by Charlton et al [18], changes from the AJCC 6th to 7th editions caused no substantial movement between stages.

Third, we did not include other potential inflammation or immune system variables that might constitute promising biomarkers in the model. Finally, an external validation of our findings has still to be performed.

\section{Conclusions}

Patients with high-grade T1 stage NMIBC with high SIM scores have worse oncologic outcomes in terms of recurrence and progression. These results could be translated into clinical practice to stratify patients who might benefit from early cystectomy.

Author contributions: Giorgio I. Russo had full access to all the data in the study and takes responsibility for the integrity of the data and the accuracy of the data analysis.
Study concept and design: Cantiello, Russo, Ferro.

Acquisition of data: Vartolomei, Abu Farhan, Terracciano, Musi, Lucarelli, Di Stasi, Hurle, Serretta, Busetto, Scafuro, Perdonà, Borghesi, Schiavina, Almeida, Bove, Lima, Ucciero, Matei, Crisan, Verze, Battaglia, Guazzoni, Autorino, Morgia, Damiano, de Cobelli, De Berardinis, Cioffi, Mirone, Shariat, Ferro.

Analysis and interpretation of data: Cantiello, Russo, Ferro.

Drafting of the manuscript: Russo.

Critical revision of the manuscript for important intellectual content: Cantiello, Ferro.

Statistical analysis: Russo.

Obtaining funding: None.

Administrative, technical, or material support: None.

Supervision: None.

Other: None.

Financial disclosures: Giorgio I. Russo certifies that all conflicts of interest, including specific financial interests and relationships and affiliations relevant to the subject matter or materials discussed in the manuscript (eg, employment/affiliation, grants or funding, consultancies, honoraria, stock ownership or options, expert testimony, royalties, or patents filed, received, or pending), are the following: None.

Funding/Support and role of the sponsor: None.

\section{Appendix A. Supplementary data}

Supplementary material related to this article can be found, in the online version, at doi:10.1016/j.euo.2018.06. 006.

\section{References}

[1] Galdiero MR, Bonavita E, Barajon I, Garlanda C, Mantovani A, Jaillon S. Tumor associated macrophages and neutrophils in cancer. Immunobiology 2013;218:1402-10.

[2] Mantovani A, Allavena P, Sica A, Balkwill F. Cancer-related inflammation. Nature 2008;454:436-44.

[3] Lucca I, Jichlinski P, Shariat SF, et al. The neutrophil-to-lymphocyte ratio as a prognostic factor for patients with urothelial carcinoma of the bladder following radical cystectomy: validation and metaanalysis. Eur Urol Focus 2016;2:79-85.

[4] Gakis G. The role of inflammation in bladder cancer. Adv Exp Med Biol 2014;816:183-96.

[5] Tang X, Du P, Yang Y. The clinical use of neutrophil-to-lymphocyte ratio in bladder cancer patients: a systematic review and metaanalysis. Int J Clin Oncol 2017;22:817-25.

[6] Pedrazzani C, Mantovani G, Fernandes E, et al. Assessment of neutrophil-to-lymphocyte ratio, platelet-to-lymphocyte ratio and platelet count as predictors of long-term outcome after $\mathrm{R} 0$ resection for colorectal cancer. Sci Rep 2017;7:1494.

[7] D’Andrea D, Moschini M, Gust K, et al. Prognostic role of neutrophilto-lymphocyte ratio in primary non-muscle-invasive bladder cancer. Clin Genitourin Cancer 2017;15:e755-64.

[8] Hendricksen K, Aziz A, Bes P, et al. Discrepancy between European Association of Urology guidelines and daily practice in the management of non-muscle-invasive bladder cancer: results of a European survey. Eur Urol Focus 2017. http://dx.doi.org/10.1016/j.euf.2017.09. 002, pii: S2405-4569(17)30206-7.

[9] Kamat AM, Sylvester RJ, Bohle A, et al. Definitions, end points, and clinical trial designs for non-muscle-invasive bladder cancer: 
recommendations from the International Bladder Cancer Group. J Clin Oncol 2016;34:1935-44.

[10] Babjuk M, Bohle A, Burger M, et al. EAU guidelines on non-muscleinvasive urothelial carcinoma of the bladder: update 2016. Eur Urol 2017;71:447-61.

[11] Li DY, Hao XY, Ma TM, Dai HX, Song YS. The prognostic value of platelet-to-lymphocyte ratio in urological cancers: a meta-analysis. Sci Rep 2017;7:15387.

[12] Mbeutcha A, Shariat SF, Rieken M, et al. Prognostic significance of markers of systemic inflammatory response in patients with nonmuscle-invasive bladder cancer. Urol Oncol 2016;34, 483.e17-24.

[13] Yoshida T, Kinoshita H, Yoshida K, et al. Prognostic impact of perioperative lymphocyte-monocyte ratio in patients with bladder cancer undergoing radical cystectomy. Tumour Biol 2016;37:10067-74

[14] Tanaka N, Kikuchi E, Kanao K, et al. Impact of combined use of bloodbased inflammatory markers on patients with upper tract urothelial carcinoma following radical nephroureterectomy: proposal of a cumulative marker score as a novel predictive tool for prognosis. Eur Urol Focus 2015;1:54-63.

[15] Wei Y, Jiang YZ, Qian WH. Prognostic role of NLR in urinary cancers: a meta-analysis. PLoS One 2014;9:e92079.

[16] Viers BR, Boorjian SA, Frank I, et al. Pretreatment neutrophil-tolymphocyte ratio is associated with advanced pathologic tumor stage and increased cancer-specific mortality among patients with urothelial carcinoma of the bladder undergoing radical cystectomy. Eur Urol 2014;66:1157-64.

[17] Templeton AJ, Knox JJ, Lin X, et al. Change in neutrophil-to-lymphocyte ratio in response to targeted therapy for metastatic renal cell carcinoma as a prognosticator and biomarker of efficacy. Eur Urol 2016;70:358-64.

[18] Charlton ME, Adamo MP, Sun L, Deorah S. Bladder cancer collaborative stage variables and their data quality, usage, and clinical implications: a review of SEER data, 2004-2010. Cancer 2014;120 (Suppl 23):3815-25. 